\title{
A novel branch-and-bound algorithm for the chance-constrained resource-constrained project scheduling problem
}

\author{
Morteza Davari $^{\mathrm{a} *}$ and Erik Demeulemeester ${ }^{\mathrm{a}}$ \\ ${ }^{a}$ Department of Decision Sciences and Information Management, KU Leuven, Leuven, Belgium
}

(Received 00 Month 20XX; accepted 00 Month 20XX)

\begin{abstract}
The resource-constrained project scheduling problem (RCPSP) has been widely studied during the last few decades. In real-world projects, however, not all information is known in advance and uncertainty is an inevitable part of these projects. The chance-constrained resource-constrained project scheduling problem (CC-RCPSP) has been recently introduced to deal with uncertainty in the RCPSP. In this paper, we propose a branch-and-bound (B\&B) algorithm and a mixed integer linear programming (MILP) formulation that solve a sample average approximation of the CC-RCPSP. We introduce two different branching schemes and eight different priority rules for the proposed B\&B algorithm. The computational results suggest that the proposed branch-and-bound procedure clearly outperforms both a proposed MILP formulation and a branch-and-cut algorithm from the literature.
\end{abstract}

Keywords: PROJECT SCHEDULING; BRANCH AND BOUND; UNCERTAINTY; CHANCE CONSTRAINTS; RESOURCE CONSTRAINTS;

\section{Introduction}

Real-life projects contain considerable levels of uncertainty. The uncertainty can be the result of many internal and external unexpected or expected events in the execution of a project. This uncertainty may lead to disruptions and may often make any schedule obtained by solving any deterministic project scheduling problem unreliable.

In order to incorporate uncertainty into the resource-constrained project scheduling problem (RCPSP) and obtain robust solutions, Lamas and Demeulemeester (2016) introduce the chanceconstrained RCPSP (CC-RCPSP) in which the makespan is minimized while the actual executed schedule is identical to the planned schedule with a certain minimum probability. In other words, the idea behind CC-RCPSP is to find a proactive solution that is as short as possible and needs no reaction with a certain minimum required probability. This probability, which is usually given by the managerial team, will be defined as the confidence level in the remainder of this paper.

In this paper, we introduce a novel branch-and-bound (B\&B) algorithm that can solve instances of a sample average approximation of the CC-RCPSP until optimality. The novelty of our B\&B algorithm is reflected in its branching schemes. In these branching schemes, we use the notion of casets that will be introduced and defined in Section 5.1. The remainder of this paper is structured as follows. First, the state of the art around the topic is provided in Section 2. Then in Section 3 a compact description of the CC-RCPSP is given. Next, both a mixed integer linear programming (MILP) formulation and a B\&B algorithm that optimally solve instances of the sample average approximation of the CC-RCPSP are proposed in Section 4 and Section 5, respectively. After that,

*Corresponding author. Email: morteza.davari@kuleuven.be 
computational results are reported in Section 6. Finally, a summary and a conclusion are given in Section 7 .

\section{Literature review}

In this section, we review the state of the art around the topic of the CC-RCPSP in three parts. In the first part (Section 2.1), the literature on the deterministic RCPSP is presented. In the second part (Section 2.2), we list a number of papers dealing with the RCPSP under uncertainty. And finally in the last part (Section 2.3), a brief review of the literature on chance-constrained programming $(\mathrm{CCP})$ is given.

\subsection{The deterministic RCPSP}

The deterministic RCPSP is known to be NP-hard in the strong sense (Blazewicz et al. 1983). However, a number of relatively efficient MILP formulations as well as other exact methods, such as B\&B and branch-and-cut (B\&C) algorithms, have been presented to solve this NP-hard problem (Demeulemeester and Herroelen 2002). Many MILP formulations for the deterministic RCPSP have been proposed. Among these, we refer to Klein (2000) who gathers six different MILP formulations for the deterministic RCPSP. Also recently, other MILP formulations have been introduced, for which we refer to Artigues et al. (2008) and Koné et al. (2011). Among many exact algorithms that are proposed during the last few decades, B\&B algorithms (Demeulemeester and Herroelen 1997; Mingozzi et al. 1998; Sprecher 2000) are the most successful approaches to optimally solve small and medium-sized instances of the deterministic RCPSP.

\subsection{The RCPSP under uncertainty}

As already motivated in the introduction, handling uncertainty in project scheduling is of great importance. The CC-RCPSP, which is the main topic of this paper and which is described in Section 3, is one of the existing problems that incorporates uncertainty in project scheduling. Besides CC-RCPSP, there are two other main streams of research dealing with RCPSP in the presence of activity duration uncertainty: the stochastic RCPSP and the proactive and reactive RCPSP.

The first trend in the literature focuses on the stochastic RCPSP which is also referred to as SRCPSP. The SRCPSP considers stochastic activity durations while minimizing the makespan. A solution in the SRCPSP is a policy which is a set of decision rules that dictate the starting of certain activities at certain decision moments in a dynamic fashion (Stork 2000). The SRCPSP is already known for many decades. It all started when Möhring and Radermacher (1985) provided an introduction for stochastic project scheduling problems. Since finding a globally optimal policy was not tractable except until recently (Creemers 2015), a large body of research is devoted on searching over different classes of policies (Stork 2001; Rostami et al. 2016).

The second trend in the literature focuses on the proactive and reactive resource-constrained project scheduling problem (PR-RCPSP). The idea of proactive and reactive scheduling is to generate a baseline schedule that is as robust as possible against disruptions (which is referred to as proactive scheduling) and to propose a reactive policy that determines how to react to possible disruptions (which is referred to as reactive scheduling) (Van de Vonder et al. 2006). A very crucial disadvantage of the SRCPSP is that no baseline schedule is constructed. This disadvantage of the SRCPSP was the trigger for the initial steps towards PR-RCPSP in which a stable baseline schedule is generated. A number of justifications for the necessity of a baseline schedule have been discussed by several authors (Demeulemeester and Herroelen 2011). Among many research papers that study 
the PR-RCPSP, we cite the recent papers by Davari and Demeulemeester (2017), Deblaere et al. (2011a,b), and Van de Vonder et al. (2008).

There are also a number of papers that do not completely fit into the two above-mentioned main streams. Bruni et al. (2011a) study a variant of the stochastic RCPSP and propose a heuristic algorithm in which a subproblem containing joint probabilistic constraints (chance-constraints) is solved to determine the set of jobs to start at each decision moment and Bruni et al. (2011b) propose a stochastic dynamic generation scheme in which a subproblem containing joint probabilistic constraints is solved to generate the activity completion times at each iteration. We also cite Artigues et al. (2013) who propose a problem to minimize absolute-regret robust RCPSP, which is modeled as an optimization problem with two-stage variables (an early-start policy and a vector of starting times), Bruni et al. (2017) who propose a two-stage optimization problem to minimize the worse-case makespan and finally Bruni et al. (2018) who propose a two-stage optimization problem to minimize the expected makespan. Similarly to the type of formulation that is used in this paper, Bruni et al. (2017, 2018) also base their models on the resource-flow formulation introduced by Artigues et al. (2003).

\subsection{Chance-constrained programming}

To the best of our knowledge, the first research on CCP problems with discrete random rhs vector is the one studied by Prékopa (1990). Dentcheva et al. (2000) use the concept of a p-efficient point of a probability distribution to derive various equivalent problems and eventually obtain lower and upper bounds for the optimal value of probabilistically constrained stochastic programming problems with discrete random variables. Ruszczyński (2002) proposes a mixed integer programming formulation together with some valid inequalities for a CCP problem with a rhs vector. Beraldi and Ruszczyński (2002) and Beraldi and Ruszczyński (2005) propose a B\&B and a beam search adaptation of the $\mathrm{B} \& \mathrm{~B}$ to deal with the $\mathrm{CCP}$ problem with a rhs vector.

Recently, a number of advancements has been done to solve CCP problems more efficiently. Stronger facet-defining valid inequalities are proposed by Luedtke et al. (2010) and Küçükyavuz (2012), an Express (Explore and Repair Stochastic Solution) heuristic is introduced by Bruni et al. (2013) and a B\&C algorithm is developed by Luedtke (2014). Also, a cell-and-bound algorithm to identify an exact solution to CCP problems with discrete distributions is proposed by Zheng et al. (2017).

\section{Problem description}

We are given a set $N=\{0,1, \ldots, n+1\}$ of activities where activities 0 and $n+1$ are the dummy start and dummy end activities, respectively. Each activity $i \in N^{\prime}=N \backslash\{0, n+1\}$ has a stochastic non-negative integer duration $\tilde{p}_{i}$, with $p_{i}^{\min } \leq \tilde{p}_{i} \leq p_{i}^{\max }$, which follows a discrete distribution $\operatorname{dist}\left(\tilde{p}_{i}\right)$. We assume that these stochastic durations are independently distributed. Notice that the durations of the dummy activities are not stochastic $\left(\tilde{p}_{0}=\tilde{p}_{n+1}=0\right)$. We are also given a set $\mathcal{R}$ of renewable resource types. Each job $i$ requires $r_{i k}$ units of resource type $k \in \mathcal{R}$ during its processing time and the resource availability of resource type $k$ is denoted by $R_{k}$. The set $E \in\{(i, j) \mid i, j \in N\}$ defines precedence constraints among the activities where the pair $(i, j) \in E$ indicates that activity $j$ cannot be started before activity $i$ is completed.

Let us define variables $x_{i j}$ that equal one if activity $i$ is completed before the start of activity $j$ and zero otherwise. We also introduce the variables $f_{i j k}$ which represent the amount of resource type $k$ that is passed from activity $i$ to activity $j$. Finally, let $\mathbf{s}$, which is a vector of non-negative starting times of the activities, be a solution for the RCPSP and $\hat{\mathbf{p}}$ be its vector of deterministic activity durations. The resource-flow formulation proposed by Artigues et al. (2003) for the deterministic 
RCPSP is given as follows:

RCPSP : $\min _{\mathbf{s}} s_{n+1}$

subject to:

$$
\begin{aligned}
& x_{i j}=1 \\
& x_{i j}+x_{j i} \leq 1 \\
& f_{i j k} \leq M^{r} x_{i j} \\
& f_{0 j k} \leq r_{j k} x_{0 j} \\
& f_{i(n+1) k} \leq r_{i k} x_{i(n+1)} \\
& \sum_{j \in N} f_{0 j k}=R_{k} \\
& \sum_{j \in N} f_{i j k}=r_{i k} \\
& \sum_{j \in N} f_{j i k}=r_{i k} \\
& s_{j}-s_{i}-M^{p}\left(x_{i j}-1\right) \geq \hat{p}_{i} \\
& \mathbf{s} \in \mathbb{N}^{n+2}, \mathbf{x} \in\{0,1\}^{(n+2)^{2}}
\end{aligned}
$$$$
\forall(i, j) \in E
$$$$
\forall(i, j) \in N^{2}, i \neq j
$$$$
\forall(i, j) \in N^{\prime 2}, i \neq j, \forall k \in \mathcal{R}
$$$$
\forall(0, j) \in N^{\prime 2}, j \neq 0, \forall k \in \mathcal{R}
$$$$
\forall(i, n+1) \in N^{\prime 2}, i \neq n+1, \forall k \in \mathcal{R}
$$$$
\forall k \in \mathcal{R}
$$$$
\forall i \in N, \forall k \in \mathcal{R}
$$$$
\forall i \in N, \forall k \in \mathcal{R}
$$$$
\forall(i, j) \in N^{2}, i \neq j, \forall l=1, \ldots, m
$$

where $M^{p}=\sum_{i \in N} \hat{p}_{i}$ and $M^{r}=\min \left\{r_{i k}, r_{j k}\right\}$. In the above formulation, the set of constraints (1) enforces the precedence relations among activities. The set of constraints (2) ensures that the two variables $x_{i j}$ or $x_{j i}$ are not both one. The sets of constraints (3)-(8) represent the resource flow among activities. The set of constraints (9) guarantees the starting time of activity $j$ to be after the completion time of activity $i$ for each pair $(i, j)$ with $x_{i j}=1$.

Let $\pi(\cdot)$ be the probability that constraints $\cdot$ are satisfied and $(1-\alpha)$ be the confidence level defined by the decision maker (note that $0 \leq \alpha \leq 1$ and $\alpha$ usually takes a value very close to 0 , for example $\alpha=0.05, \alpha=0.10$ or $\alpha=0.20$, otherwise the resulting schedule is not feasible for a large number of cases). A conceptual formulation for the CC-RCPSP is given as follows:

CC-RCPSP: $\min _{\mathbf{s}} s_{n+1}$

subject to constraints (1)-(8),(10),(11) and

$\pi\left(s_{j}-s_{i}-M^{p}\left(x_{i j}-1\right) \geq \tilde{p}_{i} \quad \forall(i, j) \in N^{2}, i \neq j, \forall l=1, \ldots, m\right) \geq 1-\alpha$.

In the above formulation, constraint (12) ensures that the constraints

$s_{j}-s_{i}-M^{p}\left(x_{i j}-1\right) \geq \tilde{p}_{i}$

$$
\forall(i, j) \in N^{2}, i \neq j, \forall l=1, \ldots, m
$$

combined are not violated with a chance of $(1-\alpha)$. The CC-RCPSP is proven to be strongly NP-hard following the straightforward reduction from the RCPSP.

\subsection{A realization-based reformulation}

The vector $\tilde{\mathbf{p}}=\left(\tilde{p}_{0}, \tilde{p}_{1}, \ldots, \tilde{p}_{n+1}\right)$ can be represented by a finite supporting set $\mathfrak{P}=\left\{\mathbf{p}^{1}, \ldots, \mathbf{p}^{|\mathfrak{P}|}\right\}$ of realizations where each realization $\mathbf{p}^{l}$ represents a vector of durations $\mathbf{p}^{l}=\left(p_{0}^{l}, p_{1}^{l}, \ldots, p_{n+1}^{l}\right) \in \mathfrak{P}$. Each realization $\mathbf{p}^{l}$ occurs with a certain probability $\pi\left(\tilde{\mathbf{p}}=\mathbf{p}^{l}\right)$, which is also represented by the 
much shorter notation $\pi_{l}$. Clearly, the summation of the probabilities of all realizations equals one $\left(\sum_{\mathbf{p}^{l} \in \mathfrak{P}} \pi_{l}=1\right)$.

Constraint (12) is very difficult to tackle. Alternatively, we decide to ensure the feasibility of each solution by finding a sufficient subset $Y$ of realizations for which the solution is feasible. A subset $Y$ of realizations is called sufficient if and only if $\sum_{\mathbf{p}^{l} \in Y} \pi_{l} \geq(1-\alpha)$. Let $\mathcal{Y}_{\mathfrak{P}}$ be the set of all sufficient subsets of $\mathfrak{P}$. The CC-RCPSP can be reformulated as follows:

CC-RCPSP-R : $\min _{(Y, \mathbf{s})} s_{n+1}$

subject to constraints (1)-(8),(10),(11) and

$s_{j}-s_{i}-M^{p}\left(x_{i j}-1\right) \geq p_{i}^{Y} \quad \forall(i, j) \in N^{2}, i \neq j, \forall l=1, \ldots, m$

$\sum_{\mathbf{p}^{l} \in Y} \pi_{l} \geq(1-\alpha)$

$Y \in \mathcal{Y}_{\mathfrak{P}}$,

where $\mathbf{p}^{Y}$ is the vector of maximum durations of subset $Y$ which is computed as follows:

$$
p_{i}^{Y}=\max _{\mathbf{p}^{l} \in Y}\left\{p_{i}^{l}\right\} \quad \forall i \in N
$$

In the above formulation, constraints (13) make sure that no precedence violation occurs for any realization $\mathbf{p}^{l} \in Y$ and constraint (14) dictates a confidence level of at least $(1-\alpha)$.

\subsection{A sample average approximation}

The size of the associated finite supporting set of realizations is often too large, and thus we use a sample average approximation (SAA) technique, which is based on Monte Carlo sampling, to generate a much smaller set $\hat{\mathfrak{P}}$ of $m$ realizations which approximates the original set $\mathfrak{P}$ (note that the size $m$ influences the quality of the approximation).

We introduce the associated SAA counterpart of the CC-RCPSP (in short SAA-RCPSP) as follows:

SAA-RCPSP : $\min _{(Y, \mathbf{s})} s_{n+1}$

subject to constraints (1)-(8),(10),(11),(13) and

$$
\begin{aligned}
& \sum_{\mathbf{p}^{l} \in Y} \pi_{l} \geq(1-\hat{\alpha}) \\
& Y \in \mathcal{Y}_{\hat{\mathfrak{P}}} .
\end{aligned}
$$

where $1-\hat{\alpha}$ is the required confidence level for this counterpart problem (note that $1-\hat{\alpha}>1-\alpha$ ). In the special case where $\pi_{l}=1 / m$ for all realizations $\mathbf{p}^{l}$ in $\hat{\mathfrak{P}}$, constraint (17) can be replaced by constraint (19).

$$
|Y| \geq\lceil(1-\hat{\alpha}) \times m\rceil
$$

Let $\epsilon=\alpha-\hat{\alpha}$ and let $p^{\max }=\max _{i \in N}\left\{p_{i}^{\max }\right\}$. Following the theorems in Luedtke and Ahmed (2008), one can show that any feasible solution to an instance of SAA-RCPSP is also feasible to 
the associated instance of CC-RCPSP with a probability of $(1-\theta)$ if

$$
m \geq \frac{1}{2 \epsilon^{2}} \log \left(\frac{1}{\theta}\right)+\frac{n}{2 \epsilon^{2}} \log \left(p^{\max }\right) .
$$

For instance if $p^{\max }=30$, then $m$ must be at least 9123 to ensure that any feasible solution to an instance of SAA-RCPSP is also feasible to the associated instance of CC-RCPSP with a probability of 0.95. However, Luedtke and Ahmed (2008) also argue that this lower bound for $m$ is very conservative and one often achieves similar confidence with a much smaller $m$. In this paper, we choose $m$ between 100 and 1600 .

As the final part of this section, we pinpoint two remarks. First, because solving CC-RCPSP becomes computationally intractable, in Sections 4 and 5, we opt to solve the SAA-RCPSP, which has been shown to be a good approximation for CC-RCPSP (a supporting empirical study and discussion can be found in Lamas and Demeulemeester (2016)). However, given unlimited computational resources, the methods presented in Sections 4 and 5 can solve instances of CC-RCPSP. Second, while using the SAA technique to generate a set of realizations, generally the probability of occurrence of every single generated realization is the same as that of any other realization in that set and equals $1 / \mathrm{m}$. However, our methods are designed for a more general case where the probabilities of occurrences of realizations need not be the same.

\section{A mathematical formulation}

In this section, we introduce a MILP counterpart of the SAA-RCPSP introduced in Section 3.2. We define variables $y_{l}$ such that if $y_{l}=1$, then $\mathbf{s}$ must be feasible for realization $\mathbf{p}^{l}\left(\mathbf{p}^{l} \in Y\right)$ and if $y_{l}=0$, then the feasibility of $\mathbf{s}$ for realization $\mathbf{p}^{l}$ is not necessary $\left(\mathbf{p}^{l} \notin Y\right)$. We propose the following MILP formulation for the SAA-RCPSP:

SAA-RCPSP-MILP : $\min _{\mathbf{s}} s_{n+1}$

subject to constraints (1)-(8),(10),(11) and

$s_{j}-s_{i}-M^{p}\left(x_{i j}-1\right) \geq y_{l} p_{i}^{l}$

$$
\forall(i, j) \in N^{2}, i \neq j, \forall l=1, \ldots, m
$$

$\sum_{l=1}^{m}\left(1-y_{l}\right) \pi_{l} \leq \hat{\alpha}$

$\mathbf{y} \in\{0,1\}^{m}$

where $M^{p}=\sum_{i \in N} p_{i}^{\max }$. Constraints (21) guarantee that $\mathbf{s}$ is feasible for realization $\mathbf{p}^{l}$ if $y_{l}=1$. Finally, constraint (22) ensures that the cumulative probability of the occurrences of the realizations in the set $Y$ (realizations $\mathbf{p}^{l}$ for which $y_{l}=1$ ) is at least $(1-\hat{\alpha})$. Based on the definitions, for each feasible vector $\mathbf{y}$, we can derive an associated sufficient set of realizations $Y$. Let us introduce $\mathbf{p}^{\mathbf{y}}$ which equals its associated vector $\mathbf{p}^{Y}$. We have

$$
p_{i}^{Y}=p_{i}^{\mathbf{y}}=\max _{\mathbf{p}^{l} \in \hat{\mathfrak{P}}^{\prime}}\left\{y_{l} p_{i}^{l}\right\}=\max _{\mathbf{p}^{l} \in Y}\left\{p_{i}^{l}\right\} .
$$

The set of constraints (21) can be replaced by the following set of conceptual constraints:

$s_{j}-s_{i}-M^{p}\left(x_{i j}-1\right) \geq p_{i}^{\mathbf{y}}$

$$
\forall(i, j) \in N^{2}, i \neq j .
$$


Although constraints (24) form a nonlinear term, they can be used to understand which realizations should be included in or excluded from $Y$. An obvious finding is that in order to reduce the makespan $\left(s_{n+1}\right)$ and possibly find an optimal solution, we must choose $\mathbf{y}$ in such a way that for some activities $i$, the value $p_{i}^{\mathbf{y}}$ takes a smaller value than $p_{i}^{\max }$. This finding is the main motivation for the reformulation introduced in the following subsection.

\subsection{A stronger formulation}

In Luedtke et al. (2010), a stronger reformulation has been proposed for the general chanceconstrained optimization problem. We follow the steps to obtain a stronger reformulation for the SAA-RCPSP. Let $\boldsymbol{\delta}_{i}$ be the vector of activity durations for each realization in $\hat{\mathfrak{P}}$, sorted in nonincreasing order. In other words, $\delta_{i}^{k}$ is the $k$ th largest duration for activity $i$. In order to keep track of the realizations in each sorted vector $\boldsymbol{\delta}_{i}$, we introduce $\sigma_{i k}$ which represents the associated realization for each pair $(i, k)$ (in other words, we have $\delta_{i}^{k}=p_{i}^{\sigma_{i k}}$ ). For example, if $p_{i}^{5}$ is the second largest duration for activity $i$, then $\delta_{i}^{2}=p_{i}^{5}$ and $\sigma_{i 2}=5$. We provide a more detailed example in Example 1 in the online supplement. For each activity $i$, we define $\eta_{i} \leq m$ as the largest $k \in\{1, \ldots, m\}$ that satisfies $\sum_{s=1}^{k} \pi_{\sigma_{i s}} \leq \hat{\alpha}$. Parameter $\eta_{i}$ can be described as an upper bound on the number of realizations $\mathbf{p}^{l}$ that can be excluded from $Y\left(y_{l}=0\right)$ such that the resulting $Y$ is still sufficient and $p_{i}^{\mathbf{y}}$ is minimized. Notice that $\eta_{0}=\eta_{n+1}=0$. Thus, a stronger formulation can be obtained:

$$
\text { SAA-RCPSP-MILP2: } \min _{\mathbf{s}} s_{n+1}
$$

subject to constraints (1)-(8),(10),(11),(22),(23) and

$$
\begin{array}{ll}
s_{j}-s_{i}-M^{p}\left(x_{i j}-1\right) \geq \delta_{i}^{1}-\sum_{k=1}^{\eta_{i}}\left(\delta_{i}^{k}-\delta_{i}^{k+1}\right)\left(1-z_{i k}\right) & \forall(i, j) \in N^{2}, i \neq j \\
z_{i k}-y_{\sigma_{i k}} \geq 0 & \forall i \in N, k=1, \ldots, \eta_{i} \\
z_{i, k+1}-z_{i k} \geq 0 & \forall i \in N, k=1, \ldots, \eta_{i} \\
z_{i, \eta_{i}+1}=1 & \forall i \in N \\
z_{i k} \in\{0,1\} & \forall i \in N, k=1, \ldots, \eta_{i} .
\end{array}
$$

In the above formulation, $z_{i k}$ is zero if we decide not to consider the $k$ th largest duration for activity $i$ and one otherwise. Also if $z_{i k}=0$, then the feasibility of the resulting schedule is not guaranteed for its associated realization $\left(\sigma_{i k}\right)$ and thus we enforce $y_{\sigma_{i k}}=0$ (constraints $(26)$ ). Obviously, it would be inefficient if $z_{i k}=1$ and $z_{i, k+1}=0$. Therefore, such cases are eliminated by constraints (27). It is not very difficult to see that in no feasible schedule $z_{i, \eta_{i}+1}$ could be equal to zero and therefore the strength of the linear relaxation bound can be improved by adding constraints (28). Notice that the set of constraints (25)-(29) is a much stronger alternative for the set of constraints $(21)$.

\section{Branch-and-bound}

In this section, we propose a B\&B algorithm that solves the SAA-RCPSP. The idea is to find a schedule with minimum makespan that is feasible for at least one sufficient set of realizations. Let $Y$ denote a set of realizations and let $\mathcal{O}\left(\mathbf{p}^{Y}\right)$ be an optimization oracle that solves the RCPSP while the vector of activity durations is $\mathbf{p}^{Y}$. We define $\mathbf{s}^{Y}$ as the schedule obtained by running $\mathcal{O}\left(\mathbf{p}^{Y}\right)$. Because $\mathbf{s}^{Y}$ is feasible for the problem with $\mathbf{p}^{Y}$, it is also feasible for all realizations in $Y$. Thus, if $Y$ is a sufficient subset, then it provides a confidence level of $(1-\hat{\alpha})$. 
For each $Y$, there exists a complement set $\bar{Y}$ of realizations such that $Y \cup \bar{Y}=\hat{\mathfrak{P}}$. For each pair $(Y, \bar{Y}), Y$ is referred to as the included set and $\bar{Y}$ is referred to as the excluded set. Obviously, if $Y$ is a sufficient subset, then the cumulative probability of occurrence of the realizations in $\bar{Y}$ is smaller than or equal to $\hat{\alpha}$. Let $\Xi$ be the set of all pairs $(Y, \bar{Y})$ for which $\sum_{\mathbf{p}^{l} \in \bar{Y}} \pi_{l} \leq \hat{\alpha}$. The SAA-RCPSP can be reformulated as follows:

$$
\min _{(Y, \bar{Y}) \in \Xi} s_{n+1}^{Y}
$$

Inspired by this conceptual formulation, we aim to use a B\&B algorithm to enumerate all pairs $(Y, \bar{Y}) \in \Xi$ and find a pair $\left(Y^{*}, \bar{Y}^{*}\right)$ with the minimum corresponding makespan $\left(s_{n+1}^{Y^{*}}\right)$.

\subsection{Constructing the tree}

As we already mentioned, we aim to find the optimal pair $\left(Y^{*}, \bar{Y}^{*}\right)$ using a B\&B algorithm. Since the included set $(Y)$ and the excluded set $(\bar{Y})$ are the complement of each other, it is sufficient to only enumerate all valid excluded sets (or all valid included sets). A conventional branching scheme can be perfectly used to enumerate all excluded sets by starting from the set of all realizations and in each node/level excluding a single realization. However, we opt not to directly exclude single realizations, but instead we exclude chained sets ${ }^{1}$ (abbreviated to casets) of realizations in our novel branching schemes.

Let us introduce $C_{i}^{k}$ as the caset of realizations with the $k^{\text {th }}$ highest duration for activity $i$. The exclusion of caset $C_{i}^{k}$ from pair $(Y, \bar{Y}) \in \Xi$ results in the pair $\left(Y \backslash C_{i}^{k}, \bar{Y} \cup C_{i}^{k}\right)$. This exclusion is possible only if the resulting pair $\left(Y \backslash C_{i}^{k}, \bar{Y} \cup C_{i}^{k}\right)$ is also a member of $\Xi$.

We immediately notice that not all possible exclusions are necessarily evaluated. Only those exclusions that have a positive impact must be considered. Therefore, we introduce the notion of beneficial exclusions. The exclusion of caset $C_{i}^{k}$ from pair $(Y, \bar{Y}) \in \Xi$ is labeled beneficial if and only if it is possible and $\mathbf{p}^{Y \backslash C_{i}^{k}}<\mathbf{p}^{Y}$ (obviously if $\mathbf{p}^{Y \backslash C_{i}^{k}}=\mathbf{p}^{Y}$, then $\mathbf{s}^{Y \backslash C_{i}^{k}}=\mathbf{s}^{Y}$ and the associated exclusion is not beneficial). We assume that $\mathbf{p}<\mathbf{p}^{\prime}$ if $\exists i \in N, p_{i}<p_{i}^{\prime}$ and $\forall i^{\prime} \in N \backslash\{i\}, p_{i} \leq p_{i}^{\prime}$.

Corollary 1. The exclusion of caset $C_{i}^{k}$ from pair $(Y, \bar{Y}) \in \Xi$ is beneficial if it is possible, $\bar{Y} \cup C_{i}^{k} \neq$ $\bar{Y}$ and $\left(C_{i}^{1} \cup \ldots \cup C_{i}^{k-1}\right) \subseteq \bar{Y}$. The reverse relation does not necessarily hold.

Some casets can never be beneficially excluded. Since considering such casets is not efficient, we limit our search to only eligible casets.

Definition 1 (Eligible caset). An eligible caset is a caset that can be beneficially excluded.

We introduce set $\mathbf{C}^{E}$ as the set of all eligible casets. Let us compute $\pi\left(C_{i}^{k}\right)=\sum_{\mathbf{p}^{l} \in C_{i}^{k}} \pi_{l}$. The following theorem is derived.

Theorem 1. A caset $C_{i}^{k}$ is eligible if and only if $\sum_{s=1}^{k} \pi\left(C_{i}^{s}\right) \leq \hat{\alpha}$.

Proof. Consider the following two sets:

$$
Y_{1}=\hat{\mathfrak{P}} \backslash\left(C_{i}^{1} \cup \ldots \cup C_{i}^{k-1}\right) \text { and } Y_{2}=Y_{1} \backslash C_{i}^{k} .
$$

If the inequality $\sum_{s=1}^{k} \pi\left(C_{i}^{s}\right) \leq \hat{\alpha}$ holds, both sets must be sufficient sets. We also know that $p_{i}^{Y_{2}}<p_{i}^{Y_{1}}$. Therefore, we conclude that the exclusion of $C_{i}^{k}$ from $Y_{1}$ is beneficial and $C_{i}^{k}$ is eligible.

On the other hand, if $C_{i}^{k}$ is eligible, it can be beneficially excluded and therefore $\left(C_{i}^{1} \cup \ldots \cup C_{i}^{k-1}\right) \subseteq$ $\bar{Y}$. Since any beneficial exclusion is possible, we have $\sum_{s=1}^{k} \pi\left(C_{i}^{s}\right) \leq \hat{\alpha}$.

\footnotetext{
${ }^{1}$ These sets are named chained sets because they often relate to each other like a group of chains
} 
We define $\zeta_{i}$ as the number of eligible casets associated with activity $i$. For each activity $i$, the caset corresponding to the highest duration $\left(C_{i}^{1}\right)$ is referred to as the lead caset. Other casets that associate with the second duration, third duration, etc are referred to as the second caset, third caset, etc, respectively. For a better understanding of eligible casets and lead casets, we refer interested readers to Example 2 in the online supplement.

\subsubsection{Constructing the activity list}

In this part, we construct an activity list (AL) (note that all activities $i$ for which $\zeta_{i}=0$ are not considered in this list) that defines the order based on which activities are considered in our proposed branching schemes (that are introduced in Sections 5.1.2 and 5.1.3). We introduce different priority rules that can be used to construct such an AL. Each priority rule consists of a sorting criterion based on which the activities are sorted and (possibly) a number of tie breakers. Two example priority rules are given below.

(Rule 1) We sort activities based on the lexicographical order of the realizations in their $\sigma_{i}$ vector.

(Rule 2) We sort activities based on the decreasing order of their lead caset sizes. As the first/second/etc tie breaking rule, we consider the sizes of their second/third/etc casets.

Although the performance of these two priority rules are acceptable (see Section 6.2), better rules can be achieved by incorporating more important criteria. We propose to take three criteria into consideration while sorting the activities:

- The first criterion is the total slack (TS) of the activity. Given realization $\mathbf{p}$ and a feasible schedule $\mathbf{s}$, let $e s_{i}(\mathbf{s}, \mathbf{p})$ and $l s_{i}(\mathbf{s}, \mathbf{p})$ be the earliest and the latest starting times of activity $i$, respectively. We denote by $\varepsilon_{i}(\mathbf{s}, \mathbf{p})$ the total slack of activity $i$ for the given pair $(\mathbf{s}, \mathbf{p})$. This total slack is computed as follows: $\varepsilon_{i}(\mathbf{s}, \mathbf{p})=l s_{i}(\mathbf{s}, \mathbf{p})-e s_{i}(\mathbf{s}, \mathbf{p})$. Activities with smaller total slack values are favored to be positioned first in the list. In our experiments, we use $\mathbf{s}^{\hat{\mathfrak{F}}}$ and $\mathbf{p}^{\hat{\mathfrak{P}}}$ to compute total slacks.

- The second criterion is the number of eligible casets (NEC) associated with the activity. Those activities with smaller numbers of casets (smaller $\zeta_{i}$ ) are favored to be positioned first in the list.

- The last criterion is the influence factor (IF) of the activity. The influence factor of activity $i$, which is denoted by $\psi_{i}$, is computed as follows:

$$
\psi_{i}=\sum_{k=1}^{\zeta_{i}} \frac{p_{i}^{\max }-v\left(C_{i}^{k}\right)}{\sum_{s=1}^{k}\left|C_{i}^{s}\right|}
$$

where $v\left(C_{i}^{k}\right)$ represents the resulting duration of activity $i$ if $C_{i}^{k}$ and its corresponding earlier casets $\left(C_{i}^{k-1}, C_{i}^{k-2}\right.$,etc) are eliminated. Activities with larger influence factor values are favored to be positioned first in the list (please see Example 3 in the online supplement).

One of these three criteria is selected as the main sorting criterion. The other two criteria are exploited as the first and the second tie breakers. The question is in which order we consider these three criteria such that the performance of the $\mathrm{B} \& \mathrm{~B}$ algorithm is maximized. These criteria can be ordered in six different ways. Table 1 depicts these six different ways, each associated with a different priority rule (Rule 3,.., Rule 8). Notice that all priority rules (Rule 1 - Rule 8) share a final tie breaking rule which dictates the activity with the smaller index to be positioned first. 
Table 1.: Different priority rules obtained by different combinations of the following three criteria: total slack (TS), number of casets (NEC) and influence factor (IF). Symbol ( $\uparrow$ ) represents an ascending order and $(\downarrow)$ denotes a descending order.

\begin{tabular}{c|c:c:c} 
Priority rule & Sorting criterion & First tie breaker & Second tie breaker \\
\hline Rule 3 & TS $(\uparrow)$ & NEC $(\uparrow)$ & IF $(\downarrow)$ \\
Rule 4 & TS $(\uparrow)$ & IF $(\downarrow)$ & NEC $(\uparrow)$ \\
Rule 5 & NEC $(\uparrow)$ & TS $(\uparrow)$ & IF $(\downarrow)$ \\
Rule 6 & NEC $(\uparrow)$ & IF $(\downarrow)$ & TS $(\uparrow)$ \\
Rule 7 & IF $(\downarrow)$ & TS $(\uparrow)$ & NEC $(\uparrow)$ \\
Rule 8 & IF $(\downarrow)$ & $\operatorname{NEC~}(\uparrow)$ & TS $(\uparrow)$
\end{tabular}

\subsubsection{Branching scheme 1}

The nodes in our $\mathrm{B} \& \mathrm{~B}$ are denoted by $\mathcal{N}_{u}$ where $u$ is the index of the node, indicating the sequence in which the nodes are visited. In each node $\mathcal{N}_{u}$ (except in the root node), we decide to exclude a caset $C_{i}^{k} \in \mathbf{C}^{E}$ that is referred to as the target caset. The target caset of node $\mathcal{N}_{u}$ is denoted by $\Theta\left(\mathcal{N}_{u}\right)$. The direct father of a node $\mathcal{N}_{u}$ is the node from which it branched whereas a node's transitive father is an ancestor (i.e., father of the father (grandfather), father of the grandfather, etc) of the node. A node's set of excluded casets is the set of all target casets of the node, its direct father and all of its transitive fathers. Since each node has a one-to-one correspondence with its set of excluded casets, without loss of generality, we let both the node and its associated set of excluded casets be represented by the same notation $\mathcal{N}_{u}$. Excluding a caset is equivalent to the exclusion of all its realizations. For each node $\mathcal{N}_{u}$, the pair $\left(Y^{\mathcal{N}_{u}}, \bar{Y}^{\mathcal{N}_{u}}\right)$ is the node's associated pair in $\Xi$. Notice that a caset may be considered excluded, before being excluded itself, with the exclusion of a combination of some other casets (we refer to Example 4 in the online supplement).

Each node $\mathcal{N}_{u}$ corresponds with a set $D\left(\mathcal{N}_{u}\right)$ of effective casets. For $\mathcal{N}_{u}$, a caset $C$ is an effective caset if its exclusion from $\mathcal{N}_{u}$ is beneficial and its associated activity is positioned after the associated activity of $\Theta\left(\mathcal{N}_{u}\right)$. The former condition guarantees an improvement in the child's vector of durations whereas the latter condition prevents duplicate exclusions of casets. Since it is not efficient to exclude any ineffective caset, the target casets of the children of a node must be members of its set of effective casets (we refer to Example 5 in the online supplement).

The branching starts with the root node $\left(\mathcal{N}_{0}\right)$. The root node, which corresponds with the situation where no caset has been excluded $\left(\mathcal{N}_{0}=\emptyset\right)$, is branched into a number of child nodes, each corresponding with the exclusion of a certain caset (remember that this caset must be a member of $D\left(\mathcal{N}_{0}\right)$ and therefore should be both possible and beneficial). Each of these child nodes is then branched into its own children and so on. Backtracking happens in a node if all its children have already been visited or if its set of eligible casets is an empty set. Each node in this B\&B tree is associated with a feasible solution for SAA-RCPSP. Thus, $\mathrm{UB}^{\mathcal{N}_{u}}=s_{n+1}^{Y^{\mathcal{N}_{u}}}$ is an upper bound for the SAA-RCPSP. We denote the best upper bound found so far by UB*.

Although we avoid duplicated combinations of excluded casets by introducing the set of effective casets, it is very difficult to modify the branching scheme such that no duplicated combination of excluded realizations occurs. This difficulty stems from the fact that many casets may contain one or more common realizations. In order to clarify this branching scheme, we suggest readers to also read Example 6 in the online supplement in which the B\&B tree is searched in depth-first mode.

Some of the nodes can be dominated by computing a lower bound. In each node $\mathcal{N}_{u}$, we compute a lower bound, that is denoted by $\mathrm{LB}^{\mathcal{N}_{u}}$. This lower bound is computed as explained in the following steps:

(1) We construct $\hat{\mathcal{N}}_{u}$ which represents an extremely pessimistic case. $\hat{\mathcal{N}}_{u}$ initially contains all casets in $\mathcal{N}_{u}$. 
(2) Let $C_{i}^{k}=\Theta\left(\mathcal{N}_{u}\right)$. Add all casets $C_{i}^{k_{1}}$ with $k_{1}=k+1, \ldots, \kappa_{i}$ to $\hat{\mathcal{N}}_{u}$ where $\kappa_{i}$ is the largest integer that satisfies the following condition:

$$
\sum_{\mathbf{p}_{l} \in \bar{Y}^{\mathcal{N}_{u}} \cup\left\{C_{i}^{k+1}, \ldots, C_{i}^{\kappa_{i}}\right\}} \pi_{l} \leq \hat{\alpha}
$$

(3) Let activity $i$ be the activity associated with the current node. For each activity $i^{\prime} \in N$ that is positioned after activity $i$ in the $\mathrm{AL}$, add all casets $C_{i^{\prime}}^{k_{1}}$ with $k_{1}=1, \ldots, \kappa_{i^{\prime}}$ to $\hat{\mathcal{N}}_{u}$ where $\kappa_{i^{\prime}}$ is the largest integer that satisfies the following condition:

$$
\sum_{\mathbf{p}_{l} \in \bar{Y} \mathcal{N}_{u} \cup\left\{C_{i^{\prime}}^{1}, \ldots, C_{i^{\prime}}^{\kappa_{i^{\prime}}}\right\}} \pi_{l} \leq \hat{\alpha}
$$

(4) $\mathrm{LB}^{\mathcal{N}_{u}}=s_{n+1}^{Y^{\hat{N}} u}$.

Every node $\mathcal{N}_{u}$ in our B\&B tree is dominated if $\mathrm{LB}^{\mathcal{N}_{u}} \geq \mathrm{UB}^{*}$. Example 7 in the online supplement illustrates how to compute the introduced lower and upper bounds (notice that the above dominance rule is not considered in the tree presented in this example).

\subsubsection{Branching scheme 2}

Similarly to branching scheme 1, branching scheme 2 also branches over casets of realizations. Each node is associated with a set of excluded casets. Without loss of generality, we use the same notation $\mathcal{N}_{u}$ to represent the $u$ th node in the tree. Branching scheme 2 differs from branching scheme 1 in two major ways. Firstly, in each node of branching scheme 2, a set of target casets can be excluded (note that this set can be an empty set) instead of one single target caset. This set of target casets is denoted by $\Omega\left(\mathcal{N}_{u}\right)$. Secondly, each level of the tree is associated with a certain activity.

The branching starts with the root node. The root node is branched into a number of child nodes associated with the first activity in the AL, each corresponding with the exclusion of a certain set of casets (remember that this set can be an empty set). Let us assume that activity $i$ is the first activity in the AL. The first child node is associated with the exclusion of the set of casets $\left\{C_{i}^{1}, \ldots, C_{i}^{\zeta_{i}}\right\}$, the second child node is associated with the exclusion of the set of casets $\left\{C_{i}^{1}, \ldots, C_{i}^{\zeta_{i}-1}\right\}$, and so on. Finally, the last node is associated with the exclusion of no caset. Each of these child nodes is then branched into its own children, which are associated with the second activity in the AL, and so on. Backtracking happens in a node if all its children have already been visited, if its set of eligible casets is an empty set or if the exclusion of its target casets is infeasible.

For branching scheme $2, \mathrm{LB}^{\mathcal{N}_{u}}$ is computed based on the following steps:

(1) We construct $\hat{\mathcal{N}}_{u}$ which represents an extremely pessimistic case. $\hat{\mathcal{N}}_{u}$ initially contains all casets in $\mathcal{N}_{u}$.

(2) Let activity $i$ be the activity associated with the current node. For each activity $i^{\prime} \in N$ that is positioned after activity $i$ in the $\mathrm{AL}$, add all casets $C_{i^{\prime}}^{k_{1}}$ with $k_{1}=1, \ldots, \kappa_{i^{\prime}}$ to $\hat{\mathcal{N}}_{u}$ where $\kappa_{i^{\prime}}$ is the largest integer that satisfies the following condition:

$$
\sum_{\mathbf{p}_{l} \in \bar{Y} \mathcal{N}_{u} \cup\left\{C_{i^{\prime}}^{1}, \ldots, C_{i^{\prime}}^{\kappa_{i^{\prime}}}\right\}} \pi_{l} \leq \hat{\alpha}
$$

(3) $\operatorname{LB}^{\mathcal{N}_{u}}=s_{n+1}^{Y_{u}^{\hat{N}_{u}}}$. 
Similarly to branching scheme 1 , every node $\mathcal{N}_{u}$ in our B\&B tree is dominated if $\mathrm{LB}^{\mathcal{N}_{u}} \geq \mathrm{UB}^{*}$. Example 8 in the online supplement illustrates the details of this branching scheme (notice that the above dominance rule is also considered in the tree associated to this example).

\section{$5.2 \quad$ Improvements by hashing and listing}

Although $\mathrm{LB}^{\mathcal{N}_{u}}$ is a valid lower bound and thus can be used to prune our B\&B tree, its computation can be costly since it requires calling the optimization oracle $\mathcal{O}(\cdot)$. Therefore, one might be interested in finding a computationally much cheaper lower bounding approach. Assume $\mathbf{p}^{\hat{Y}}$ is a vector of durations for which $\mathcal{O}\left(\mathbf{p}^{\hat{Y}}\right)$ has already been solved and $\mathbf{s}^{\hat{Y}}$ is its resulting optimal solution. If $\mathbf{p}^{\hat{Y}} \leq \mathbf{p}^{Y^{\hat{N}_{u}}}$, then $s_{n+1}^{\hat{Y}} \leq \mathrm{LB}^{\mathcal{N}_{u}}$. In our B\&B algorithm, we call the optimization oracle $\mathcal{O}\left(\mathbf{p}^{Y^{\hat{N_{u}}}}\right)$ only if for each $\mathbf{p}^{\hat{Y}} \leq \mathbf{p}^{Y^{\hat{N}_{u}}}$, the inequality $s_{n+1}^{\hat{Y}}<\mathrm{UB}^{*}$ is satisfied. Otherwise, the node $\mathcal{N}_{u}$ is dominated.

In our algorithm, every time the oracle $\mathcal{O}\left(\mathbf{p}^{Y}\right)$ is solved, we store the pair $\left(\mathbf{p}^{Y}, \mathbf{s}^{Y}\right)$ both in a hash table and in a linked list. The hash table is used to avoid calling $\mathcal{O}\left(\mathbf{p}^{Y}\right)$ more than once for the nodes with a common set of excluded realizations whereas the linked list is used for the lower bound computation. Before calling the optimization oracle to compute the lower bound, we check all pairs $\left(\mathbf{p}^{Y}, \mathbf{s}^{Y}\right)$ in the linked list and ensure that no pair in the linked list is sufficient to conclude the domination of the node. The members of the linked list are constantly ordered by the number of times they successfully caused a domination.

\section{Computational results}

In this section, we report computational results for our B\&B algorithm. We also compare the performance of our B\&B algorithm with the given MILP formulation in Section 4 and the B\&C algorithm proposed by Lamas and Demeulemeester (2016). To implement the B\&B algorithm, the MILP formulation and the B\&C algorithm, Visual $\mathrm{C}++2010$ and Cplex 12.5.1 were used. All computational results were obtained on a computer with Intel(R) Xeon(R) CPU E5-2699 v3 2.30 $\mathrm{GHz}$ (2 processors, 36 cores), 256GB of RAM and running under Windows Server $2012 \mathrm{R} 2$. It is worth mentioning that in our experiments, at each time instant, 32 problem instances ran in parallel such that each instance was using only one thread (core). The remaining four cores were deliberately kept idle to deal with any possible overhead tasks and thus refrain such tasks from significantly influencing the results in our experiments.

We chose a memory limit of $10 \mathrm{~GB}$ and a time limit of one hour to solve each instance of the problem using any of the methods. The $\mathrm{B} \& \mathrm{~B}$ method and the $\mathrm{B} \& \mathrm{C}$ method usually required about a few hundreds MB of RAM (note that the required memory to solve the $\mathrm{B} \& \mathrm{C}$ algorithm is significantly larger than that of our B\&B algorithm). It is worth mentioning that, in our experiments, these two algorithms never exceeded the memory limit. The MILP formulation, on the other hand, requires a larger amount of memory which is often still less than $10 \mathrm{~GB}$. This method exceeded the memory limit only once in our experiments. If an instance is solved within the time and memory limits, it is labeled solved, and otherwise unsolved.

\subsection{Instance generation}

All methods are tested on a set of instances that are composed of the PSPLIB instances. Only instances with 30 non-dummy activities are considered in this experiment. PSPLIB is a class of instances for the deterministic RCPSP (Kolisch and Sprecher 1997), thus they need to be modified to suit our problem. The following modifications are applied on this set of instances: the activity durations $\tilde{p}_{i}$ for each non-dummy activity $i$ follow a discretized beta distribution with shape 
Table 2.: Average CPU times (in seconds) and number of solved instances within the time limit (out of 960) for different choices of priority rules and different branching schemes.

\begin{tabular}{c|c:c|c}
\multirow{2}{*}{ Priority rule } & \multicolumn{2}{|c|}{ BS1 } & BS2 \\
\cline { 2 - 4 } & Depth-first & Best-first & Best-first \\
\hline Rule 1 & $339.60(897)$ & $408.98(884)$ & $422.01(878)$ \\
Rule 2 & $347.49(895)$ & $394.20(885)$ & $389.61(883)$ \\
Rule 3 & $\mathbf{1 7 8 . 8 1 ( \mathbf { 9 2 5 } )}$ & $213.08(919)$ & $207.64(920)$ \\
Rule 4 & $211.58(917)$ & $233.99(914)$ & $238.86(910)$ \\
Rule 5 & $374.06(887)$ & $447.94(874)$ & $469.47(866)$ \\
Rule 6 & $495.57(861)$ & $624.88(831)$ & $656.77(820)$ \\
Rule 7 & $540.97(855)$ & $611.26(838)$ & $620.38(831)$ \\
Rule 8 & $536.24(856)$ & $608.88(838)$ & $615.14(836)$
\end{tabular}

parameters 2 and 5 that is mapped over the interval $\left[0.75 \hat{p}_{i}, 1.625 \hat{p}_{i}\right]$ where $\hat{p}_{i}$ is the duration of activity $i$ that is given in the original instance. In order to reduce the number of experiments, we only consider the instances from the set J30 of PSPLIB with the following filename syntax: J30X_1 $(\mathrm{X}=1, \ldots, 48)$. The random generator's seed for the instance obtained from J30X_1 equals X.

The size of the set $\mathfrak{P}$ can be extremely large. Any algorithm (including MILP solvers) that solves the problem might not be computationally tractable if $\mathfrak{P}$ is large. Therefore, we apply a sample average approximation technique to deal with the problem. We generate several sets of realizations with different sizes $(m=100,200,400,800$ or 1600) as explained in Lamas and Demeulemeester (2016). We select $1-\hat{\alpha}$ from the set $\{0.99,0.95,0.90,0.80\}$. For each combination of $(\mathrm{X}, m,(1-\hat{\alpha}))$, an instance results and thus the total number of instances is $48 \times 5 \times 4=960$.

\subsection{Overall results}

We run our B\&B algorithm on the set of instances described in Section 6.1 using different branching schemes and different priority rules. We report the overall results in Table 2. In separate experiments, we evaluate the performance of our $\mathrm{B} \& \mathrm{~B}$ algorithm for each combination of a branching scheme and a priority rule. Branching schemes that are used in these experiments are branching scheme 1 (BS1) in depth-first mode, BS1 in a best-first mode and branching scheme 2 (BS2) in a best-first mode. Notice that we deliberately decide not to include BS2 in a depth-first mode in our experiments since the order in which the sets of excluded casets are evaluated in BS2 in a depthfirst mode highly resembles that in BS1 in a depth-first mode. Also, preliminary results indicate that the required CPU time, the number of nodes and the number of oracle calls for these two approaches are very close. We use all priority rules described in Section 5.1.1 in these experiments.

Among all branching schemes, branching scheme 1 in a depth-first mode clearly performs the best. This better performance can be justified by expressing the importance of tight upper bounds. Obviously, a tight upper bound in the early stages of searching the tree can help pruning the low-quality branches. In a best-depth mode, such a tight upper bound is often obtained very late.

Our B\&B algorithm performs best when Rule 3 is used to construct the activity list. This suggests that the total slack is the most important criterion, the number of casets is the second important criterion and the influence factor is the least important criterion. It also suggests that the choice of priority rule significantly influences the performance of our B\&B algorithm.

The comparison between different settings in Table 2 is not very clear because the number of solved instances varies for different settings. In order to provide a better comparison between settings, each pair must be separately compared in more detail. In such a comparison, one should only consider the instances that are solved to optimality in the settings under question. In Table 3, we compare two different settings, namely the combination of BS1 and Rule 1 (also denoted by 
Table 3.: The average percentage deviation of the required CPU time using (BS1, Rule 3) in a depth-first mode from the required CPU time using (BS1, Rule 1) in a depth-first mode (in percentage) and the number of instances solved in both settings (out of 48) for different choices of $1-\hat{\alpha}$ and $m$.

\begin{tabular}{c|c|c:c:c:c}
\multirow{2}{*}{$1-\hat{\alpha}$} & \multicolumn{6}{|c}{$m$} \\
\cline { 2 - 6 } & 100 & 200 & 400 & 800 & 1600 \\
\hline 0.99 & $-8.91(48)$ & $-7.21(48)$ & $-39.37(48)$ & $-32.65(48)$ & $-47.94(48)$ \\
0.95 & $-42.27(48)$ & $-53.72(47)$ & $-61.07(47)$ & $-65.85(46)$ & $-78.26(44)$ \\
0.90 & $-48.64(46)$ & $-70.54(47)$ & $-71.63(46)$ & $-65.44(44)$ & $-80.60(43)$ \\
0.80 & $-69.19(45)$ & $-83.97(45)$ & $-74.74(40)$ & $-85.25(37)$ & $-94.18(30)$
\end{tabular}

the pair (BS1, Rule 1)) in a depth-first mode and (BS1, Rule 3) also in a depth-first mode. Let $\mathrm{CPU}_{I}(\mathrm{BS} 1$, Rule 1) be the CPU time required to optimally solve instance $I$ using the former setting and $\mathrm{CPU}_{I}(\mathrm{BS} 1$, Rule 3$)$ be the $\mathrm{CPU}$ time required to optimally solve instance $I$ using the latter setting. We compute the average percentage deviation of the required CPU time using (BS1, Rule 3) in a depth-first mode from the required CPU time using (BS1, Rule 1) in a depth-first mode as follows:

$$
\operatorname{avg}\left\{\frac{\mathrm{CPU}_{I}(\mathrm{BS} 1, \text { Rule } 3)-\mathrm{CPU}_{I}(\mathrm{BS} 1, \text { Rule } 1)}{\mathrm{CPU}_{I}(\mathrm{BS} 1, \text { Rule } 1)} \times 100 \%\right\} \text {. }
$$

According to the results that are presented in Table 3 , for every choice of $1-\hat{\alpha}$ and $m$, (BS1, Rule 3) performs better than (BS1, Rule 1) (negative average percent deviation). We notice that by increasing the number of realizations $(m)$ and also by decreasing the confidence level $(1-\hat{\alpha})$, the associated average percent deviation decreases. In other words, by increasing the number of realizations and also by decreasing the confidence level, the difference between the performances of (BS1, Rule 3) and (BS1, Rule 1) becomes more significant. For instance, when $1-\hat{\alpha}=0.95$ and $m=200$, (BS1, Rule 3) performs about two times faster than (BS1, Rule 1) whereas when $1-\hat{\alpha}=0.80$ and $m=1600$, (BS1, Rule 3) performs about 17 times faster than (BS1, Rule 1).

Among all combinations, (BS1, Rule 3) in a depth-first mode performs the best. In the remainder of this paper, we only report the results of our B\&B for this combination and the results associated with other combinations are ignored. Note that in the following subsections, for the sake of simplicity, we mention no setting and instead we simply use the notion 'our B\&B'.

\subsection{Detailed results}

In Table 4 , for each pair $((1-\hat{\alpha}), m)$, we report the number of instances that are solved within the time limit (Solved), the average and maximum CPU times $(\operatorname{avg}(\mathrm{CPU})$ and $\max (\mathrm{CPU}))$, the average number of casets $\left(\operatorname{avg}\left(\left|\mathbf{C}^{E}\right|\right)\right)$, the average number of nodes visited in the tree $(\operatorname{avg}(\mathrm{NN}))$ and the average number of times the optimization oracle is called $(\operatorname{avg}(\mathrm{OC}))$.

We observe that by decreasing $1-\hat{\alpha}$ from 0.99 to 0.80 and/or by increasing the number of realizations $(m)$, the average number of casets, the average CPU times, the average number of nodes and the average number of oracle calls are often increased whereas the average number of solved instances is decreased or remains unchanged.

We report that the average number of oracle calls is increased almost linearly by increasing the number of realizations, which is the main strength of this approach. However, the average number of oracle calls is increased exponentially with an increase in the number of casets (see Figure 1). 
Table 4.: The detailed computational results for our B\&B algorithm.

\begin{tabular}{|c|c|c|c|c|c|c|c|c|}
\hline \multirow{2}{*}{$1-\hat{\alpha}$} & \multirow{2}{*}{$m$} & \multirow{2}{*}{ Solved } & \multicolumn{2}{|c|}{$\mathrm{CPU}$} & \multirow{2}{*}{$\operatorname{avg}\left(\left|\mathbf{C}^{E}\right|\right)$} & & \multirow{2}{*}{$\operatorname{avg}(\mathrm{NN})$} & \multirow{2}{*}{$\operatorname{avg}(\mathrm{OC})$} \\
\hline & & & avg & $\max$ & & & & \\
\hline \multirow{5}{*}{0.99} & 100 & 48 & 2.71 & 118.56 & 6.94 & 1 & 6.73 & 7.73 \\
\hline & 200 & 48 & 3.29 & 133.55 & 10.38 & 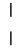 & 16.33 & 14.40 \\
\hline & 400 & 48 & 18.95 & 859.73 & 14.46 & 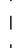 & 45.38 & 28.83 \\
\hline & 800 & 48 & 22.76 & 943.68 & 17.56 & ! & 136.98 & 66.63 \\
\hline & 1600 & 48 & 72.04 & 2994.27 & 21.10 & & 243.94 & 84.71 \\
\hline \multirow{5}{*}{0.95} & 100 & 48 & 32.61 & 1360.92 & 22.19 & 1 & 113.96 & 71.73 \\
\hline & 200 & 48 & 60.56 & 2611.33 & 26.02 & I & 327.60 & 161.38 \\
\hline & 400 & 47 & 95.85 & 3600.00 & 30.35 & । & 1076.27 & 380.25 \\
\hline & 800 & 46 & 171.39 & 3600.00 & 33.98 & I & 3031.90 & 825.19 \\
\hline & 1600 & 46 & 206.83 & 3600.00 & 37.19 & 1 & 5484.21 & । 1290.60 \\
\hline \multirow{5}{*}{0.90} & 100 & 47 & 101.44 & 3600.00 & 31.75 & I & 732.35 & 358.17 \\
\hline & 200 & 47 & 110.79 & 3600.00 & 36.42 & 1 & 1893.04 & 729.15 \\
\hline & 400 & 46 & 184.42 & 3600.00 & 40.46 & I & 5725.63 & 1586.52 \\
\hline & 800 & 46 & 263.40 & 3600.00 & 44.27 & 1 & 18093.48 & 4176.90 \\
\hline & 1600 & 45 & 296.78 & 3600.00 & 48.19 & 1 & 26253.33 & 5392.23 \\
\hline \multirow{5}{*}{0.80} & 100 & 46 & 258.06 & 3600.00 & 44.42 & & 6028.85 & 2270.23 \\
\hline & 200 & 46 & 212.91 & 3600.00 & 49.08 & I & 17682.98 & 4882.92 \\
\hline & 400 & 43 & 402.35 & 3600.00 & 53.58 & । & 39607.83 & 9144.67 \\
\hline & 800 & 42 & 509.95 & : 3600.00 & 57.79 & & 136760.71 & 24427.54 \\
\hline & 1600 & 42 & 549.09 & 3600.00 & 61.98 & I & 156366.33 & 27565.60 \\
\hline
\end{tabular}

Figure 1.: Logarithm of number of oracle calls vs. number of casets.

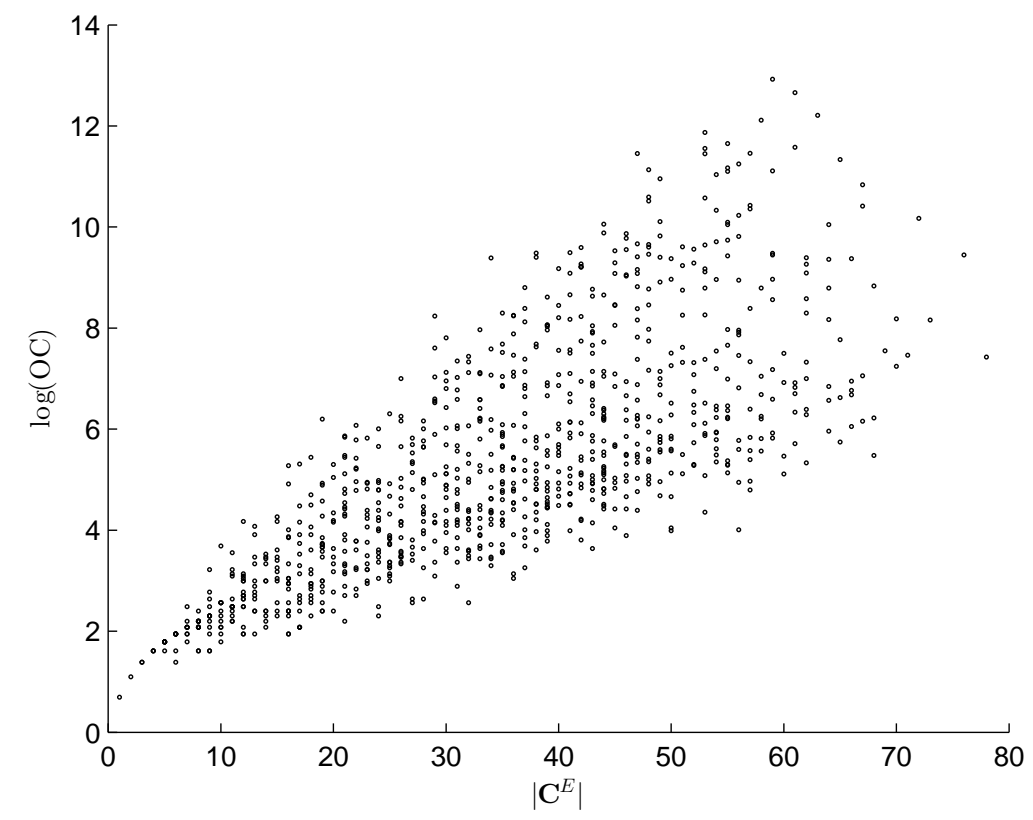

\subsubsection{Quality of the lower bound}

One of the main features of our B\&B algorithm is the lower bound computation. To show the strength of the proposed lower bound, we report the average deviation of the lower bound in the 
Table 5.: The average percent deviation between the lower bound and the objective value of the best found (or optimal) solution for different choices of $1-\hat{\alpha}$ and $m$.

\begin{tabular}{c|c:c:c:c:c}
\multirow{2}{*}{$1-\hat{\alpha}$} & \multicolumn{7}{|c}{$m$} \\
\cline { 2 - 6 } & 100 & 200 & 400 & 800 & 1600 \\
\hline 0.99 & -2.23 & -2.63 & -3.16 & -3.26 & -3.17 \\
0.95 & -5.86 & -5.71 & -6.28 & -6.12 & -6.35 \\
0.90 & -7.68 & -8.39 & -8.15 & -8.25 & -8.25 \\
0.80 & -10.69 & -11.31 & -11.06 & -11.44 & -11.67
\end{tabular}

Table 6.: The effect of implementing the hash table (HT) and/or the linked list (LL) on our B\&B algorithm.

\begin{tabular}{c|c:c:c} 
Setting & $\begin{array}{c}\text { Solved (out of } \\
960)\end{array}$ & $\operatorname{avg}(\mathrm{CPU})$ & $\operatorname{avg}(\mathrm{OC})$ \\
\hline B\&B & 925 & 178.81 & 4173.27 \\
B\&B - HT & 925 & 180.81 & 4514.47 \\
B\&B - LL & 913 & 239.61 & 19483.30 \\
B\&B - HT - LL & 913 & 241.19 & 19686.32
\end{tabular}

root node from the objective value of the best found (optimal) solution in Table 5 . This average deviation is computed as follows:

$$
\operatorname{avg}\left\{\frac{\mathrm{LB}^{\mathcal{N}_{0}}-U B^{*}}{U B^{*}} \times 100 \%\right\} .
$$

Notice that the computation of the lower bound in the root node is exactly the same for the two branching schemes. The results in Table 5 suggest that by decreasing the confidence level and by increasing the number of realizations, the quality of the lower bound is generally decreased.

\subsubsection{Impacts of hashing and listing}

In Section 5.2, we discussed two improvement techniques, namely exploiting a hash table (HT) and a linked list (LL). Table 6 demonstrates their effects on the performance of our B\&B algorithm. The first row represents the setting in which both the hash table and the linked list are exploited. The second and third row represent the settings in which either of the two improving techniques is not exploited. Finally, the last low represents the setting in which none of them is exploited. Based on these results, we notice that implementing the hash table slightly improves the performance of our B\&B algorithm whereas the improvement resulting from the implementation of the linked list is significant.

\subsubsection{Results for instances with a large number of realizations}

Readers may be interested in the performance of our B\&B algorithm when larger sets of realizations are considered. In this part, we present the results of an additional set of instances. This set is generated similarly to the set of instances introduced in Section 6.1, except that $m=3200,6400,12800,25600$ or 51200 . Table 7 reports the average CPU times and the numbers of instances solved for different choices of $1-\hat{\alpha}$ and these large $m$ values. We notice that our B\&B algorithm can solve 35 (out of 48) instances even for the most difficult setting, i.e., the setting where $m=51200$ and $1-\hat{\alpha}=0.80$. The main reason of such a good performance lies in the way the casets are introduced. Having a fixed range of integer numbers, the number of casets remains almost constant by increasing the number of realizations. Note that the number of oracle 
Table 7.: The average CPU time and the number of instances solved (out of 48) for different choices of $1-\hat{\alpha}$ and large $m$ values.

\begin{tabular}{|c|c|c|c|c|c|}
\hline \multirow{2}{*}{$1-\hat{\alpha}$} & \multicolumn{5}{|c|}{$m$} \\
\hline & 3200 & 6400 & 12800 & 25600 & 51200 \\
\hline 0.99 & $35.66(48)$ & $\begin{array}{l}92.69(48) \\
\end{array}$ & $134.54(47)$ & $\begin{array}{ll} & 160.03(47)\end{array}$ & $245.81(45)$ \\
\hline 0.95 & $247.56(45)$ & $347.23(44)$ & $388.62(45)$ & $381.67(44)$ & $413.95(44)$ \\
\hline 0.90 & $406.65(44)$ & $484.43(44)$ & $517.01(42)$ & $675.13(41)$ & $737.77(40)$ \\
\hline 0.80 & $697.72(40)$ & $846.37(39)$ & $946.33(38)$ & $1029.67(36)$ & $1037.80(35)$ \\
\hline
\end{tabular}

Table 8.: The detailed computational results for our B\&B algorithm ran on instances with medium variances.

\begin{tabular}{|c|c|c|c|c|c|c|c|}
\hline \multirow{2}{*}{$1-\hat{\alpha}$} & \multirow{2}{*}{$m$} & \multirow{2}{*}{ Solved } & \multicolumn{2}{|c|}{$\mathrm{CPU}$} & \multirow{2}{*}{$\operatorname{avg}\left(\left|\mathbf{C}^{E}\right|\right)$} & \multirow{2}{*}{$\operatorname{avg}(\mathrm{NN})$} & \multirow{2}{*}{$\operatorname{avg}(\mathrm{OC})$} \\
\hline & & & avg & $\max$ & & & \\
\hline \multirow{5}{*}{0.99} & 100 & 48 & 3.99 & 166.937 & 13.15 & 12.52 & 13.50 \\
\hline & 200 & 48 & 22.70 & 1007.54 & 20.77 & 45.50 & 41.33 \\
\hline & 400 & 48 & 89.79 & : 3490.83 & 29.19 & 238.63 & 184.52 \\
\hline & 800 & 47 & 120.67 & 3600.00 & 36.29 & 1274.45 & 659.21 \\
\hline & 1600 & 46 & 249.27 & 3600.00 & 42.42 & 6458.27 & 2583.79 \\
\hline \multirow{5}{*}{0.95} & 100 & 47 & 109.14 & 3600.00 & 42.06 & 835.25 & 628.56 \\
\hline & 200 & 45 & 313.76 & 3600.00 & 52.75 & 5612.54 & 2950.17 \\
\hline & 400 & 44 & 480.87 & 3600.00 & 62.13 & । 54531.02 & 19719.38 \\
\hline & 800 & 39 & 842.35 & 3600.00 & 69.25 & 218721.46 & 50665.90 \\
\hline & 1600 & 36 & 1178.17 & 3600.00 & 75.50 & | 399935.52 & 85520.31 \\
\hline \multirow{5}{*}{0.90} & 100 & 45 & 313.66 & 3600.00 & 61.23 & 12494.23 & 6862.81 \\
\hline & 200 & 42 & 570.21 & 3600.00 & 73.31 & 74333.40 & 27812.06 \\
\hline & 400 & 34 & 1274.74 & 3600.00 & 82.67 & | 324515.38 & 86804.75 \\
\hline & 800 & 33 & 1294.26 & 3600.00 & 91.17 & 441609.19 & 92741.42 \\
\hline & 1600 & 28 & 1582.64 & 3600.00 & 97.98 & । 576703.73 & 115854.06 \\
\hline \multirow{5}{*}{0.80} & 100 & 38 & 903.09 & 3600.00 & 85.13 & । 175219.60 & 67898.67 \\
\hline & 200 & 34 & 1266.76 & 3600.00 & 97.88 & 330257.13 & 98129.88 \\
\hline & 400 & 29 & 1591.19 & 3600.00 & 108.33 & 522442.42 & 126655.79 \\
\hline & 800 & 27 & 1700.57 & 3600.00 & 116.96 & 653758.15 & 128094.58 \\
\hline & 1600 & 24 & 1905.06 & 3600.00 & 124.79 & । 935444.83 & 167067.04 \\
\hline
\end{tabular}

calls and as such the required CPU times are exponentially increased only by increasing the number of casets, as it has been shown in Figure 1. Therefore, because the number of casets remains almost constant by increasing the number of realizations, we conclude that increasing the number of realizations does not lead to a significant increase in CPU times.

\subsubsection{Results for instances with medium and high variances}

The activity durations of the instances introduced in Section 6.1 are generated from the range $\left[0.75 \hat{p}_{i}, 1.625 \hat{p}_{i}\right]$ which represents a low variance. In most papers that are dealing with project scheduling where activity durations are stochastic, two wider ranges are also used: a range of $\left[0.5 \hat{p}_{i}, 2.25 \hat{p}_{i}\right]$ which represents a medium variance in activity durations and a range of $\left[0.25 \hat{p}_{i}, 2.875 \hat{p}_{i}\right]$ which represents a high variance in activity durations. We introduce two additional sets of instances that are generated similarly to the set of instances introduced in Section 6.1, except that the medium and the high variance ranges are used to generate the activity durations. Tables 8 and 9 show the detailed computational results for our B\&B algorithm ran on instances 
Table 9.: The detailed computational result for our B\&B algorithm ran on instances with high variances.

\begin{tabular}{|c|c|c|c|c|c|c|c|}
\hline \multirow{2}{*}{$1-\hat{\alpha}$} & \multirow{2}{*}{$m$} & \multirow{2}{*}{ Solved } & \multicolumn{2}{|c|}{$\mathrm{CPU}$} & \multirow{2}{*}{$\operatorname{avg}\left(\left|\mathbf{C}^{E}\right|\right)$} & \multirow{2}{*}{$\operatorname{avg}(\mathrm{NN})$} & \multirow{2}{*}{ avg $(\mathrm{OC})$} \\
\hline & & & avg & $\max$ & & & \\
\hline \multirow{5}{*}{0.99} & 100 & 48 & 11.08 & 502.98 & 15.31 & 14.42 & 15.35 \\
\hline & 200 & 48 & 29.25 & | 1189.15 & 26.15 & 68.00 & 65.52 \\
\hline & 400 & 47 & 141.76 & : 3600.00 & 39.67 & 576.04 & 495.94 \\
\hline & 800 & 45 & 319.38 & 3600.00 & 50.75 & 4691.56 & 2697.77 \\
\hline & 1600 & 43 & 442.91 & 3600.00 & 62.40 & 26714.71 & 10894.48 \\
\hline \multirow{5}{*}{0.95} & 100 & 47 & 131.68 & 3600.00 & 53.27 & 1199.21 & 965.15 \\
\hline & 200 & 43 & 416.64 & 3600.00 & 70.67 & 12984.54 & 7192.79 \\
\hline & 400 & 36 & 957.54 & : 3600.00 & 87.29 & 162026.98 & 63291.98 \\
\hline & 800 & 31 & 1404.69 & 3600.00 & 100.33 & 357048.48 & 104380.56 \\
\hline & 1600 & 27 & 1632.23 & 3600.00 & 112.33 & । 529853.10 & I 126483.63 \\
\hline \multirow{5}{*}{0.90} & 100 & 43 & 470.28 & 3600.00 & 81.10 & 20620.33 & । 13079.69 \\
\hline & 200 & 36 & 1053.60 & 3600.00 & 100.50 & 191257.79 & 82103.69 \\
\hline & 400 & 27 & 1769.10 & 3600.00 & 117.21 & । 427891.35 & । 141403.94 \\
\hline & 800 & 25 & 1880.22 & 3600.00 & 129.98 & 615474.25 & 158701.44 \\
\hline & 1600 & 21 & 2274.00 & 3600.00 & 142.96 & 851616.31 & I 183599.63 \\
\hline \multirow{5}{*}{0.80} & 100 & 32 & 1408.39 & 3600.00 & 118.40 & 227325.42 & 109373.13 \\
\hline & 200 & 22 & 2090.18 & 3600.00 & 138.65 & 462194.48 & 170729.17 \\
\hline & 400 & 18 & 2440.46 & 3600.00 & 155.69 & 645457.58 & 195175.23 \\
\hline & 800 & 16 & 2653.74 & $\begin{array}{l}3600.00 \\
360\end{array}$ & 169.06 & | 910299.63 & 216549.46 \\
\hline & 1600 & 13 & 2892.69 & 3600.00 & 181.88 & 1111028.08 & 232467.46 \\
\hline
\end{tabular}

with medium and high variances, respectively. As expected, by increasing the range (variance), the number of casets and as such the CPU times are increased whereas the number of solved instances is decreased. It is interesting to see that our B\&B algorithm can also solve most of the instances with medium variance and a reasonably large number of instances with high variance to optimality within the time limit.

\subsection{Comparison with other methods}

We compare our B\&B algorithm with the mathematical formulations proposed in Section 4 and the B\&C algorithm proposed by Lamas and Demeulemeester (2016). For each pair $(1-\hat{\alpha}, m)$, Table 10 reports the number of solved instances within different time limits: 10 seconds (10s), 1 minute $(1 \mathrm{~m}), 10$ minutes $(10 \mathrm{~m})$ and 1 hour $(1 \mathrm{~h})$. We observe that our B\&B algorithm clearly outperforms the mathematical formulation and the $\mathrm{B} \& \mathrm{C}$ algorithm in all settings.

As we decrease $(1-\hat{\alpha})$, the number of solved instances is decreased for all three methods. The same behavior is noticed when we increase the number of realizations. Intriguingly we notice that within 10 seconds, our $\mathrm{B} \& \mathrm{~B}$ algorithm can solve more instances than the number of instances that are solved within one hour by the mathematical formulation. Also within one minute, our $\mathrm{B} \& \mathrm{~B}$ algorithm can solve more instances than the number of instances that are solved within one hour using the $\mathrm{B} \& \mathrm{C}$ algorithm. More interestingly, in many very difficult settings (for example the setting where $(1-\hat{\alpha})=0.80$ and $m=1600)$, the number of instances that are solved within 10 seconds by our B\&B algorithm is much more than the number of instances that are solved within one hour using the $\mathrm{B} \& \mathrm{C}$ algorithm.

As is also clear in the table, $\mathrm{B} \& \mathrm{C}$ is generally performing better than the MILP formulation. However, when $1-\hat{\alpha} \leq 0.90$ and $m \geq 400$ the MILP formulation sometimes performs better, 
Table 10.: The number of instances solved to optimality for different time limits (10 seconds, 1 minute, 10 minutes and 1 hour), different methods and different choices of $1-\hat{\alpha}$ and large $m$ values.

\begin{tabular}{|c|c|c|c|c|c|c|c|c|c|c|c|c|c|}
\hline \multirow{2}{*}{$1-\hat{\alpha}$} & \multirow{2}{*}{$m$} & \multicolumn{4}{|c|}{$B \& B$} & \multicolumn{4}{|c|}{ SAA-RCPSP-MILP2 } & \multicolumn{4}{|c|}{$\mathrm{B} \& \mathrm{C}$} \\
\hline & & $10 \mathrm{~s}$ & $1 \mathrm{~m}$ & $10 \mathrm{~m}$ & $1 \mathrm{~h}$ & $10 \mathrm{~s}$ & $1 \mathrm{~m}$ & $10 \mathrm{~m}$ & $1 \mathrm{~h}$ & $10 \mathrm{~s}$ & $1 \mathrm{~m}$ & $10 \mathrm{~m}$ & $1 \mathrm{~h}$ \\
\hline \multirow{5}{*}{0.99} & 100 & 47 & 47 & 48 & 48 & 26 & 30 & 33 & 38 & 39 & 44 & 47 & 47 \\
\hline & 200 & 47 & 47 & 48 & 48 & 26 & 30 & 32 & 35 & 38 & 43 & 47 & 47 \\
\hline & 400 & 46 & 47 & 47 & 48 & 24 & 29 & 32 & 36 & 38 & 40 & 46 & 46 \\
\hline & 800 & 43 & 46 & 47 & 48 & 22 & 28 & 31 & 34 & 33 & $\begin{array}{l}39 \\
3\end{array}$ & 43 & 46 \\
\hline & 1600 & 43 & 45 & 47 & 48 & 19 & 30 & 32 & 35 & 28 & 37 & 42 & 46 \\
\hline \multirow{5}{*}{0.95} & 100 & 44 & 46 & 47 & 48 & 21 & 30 & 30 & 37 & 35 & 39 & 43 & 46 \\
\hline & 200 & 42 & 45 & 47 & 48 & 19 & 30 & 31 & 36 & 30 & 39 & 42 & 44 \\
\hline & 400 & 41 & 43 & 47 & 47 & 18 & 29 & 32 & 36 & 24 & 34 & 40 & 43 \\
\hline & 800 & 42 & 43 & 45 & 46 & 9 & 24 & 32 & 37 & 16 & 27 & 38 & 40 \\
\hline & 1600 & 41 & 43 & 44 & 46 & 6 & 25 & 29 & 32 & 6 & 22 & 31 & 39 \\
\hline \multirow{5}{*}{0.90} & 100 & 41 & 44 & 46 & 47 & 17 & 28 & 31 & 37 & 28 & $\begin{array}{l}y 38 \\
138\end{array}$ & \begin{tabular}{|l} 
\\
\end{tabular} & 44 \\
\hline & 200 & 42 & 42 & 46 & 47 & 10 & 25 & 31 & 35 & 23 & 32 & 38 & 42 \\
\hline & 400 & 40 & 43 & 45 & 46 & 8 & 23 & 31 & 33 & 12 & 26 & 38 & 40 \\
\hline & 800 & 40 & 41 & 43 & 46 & 5 & 19 & 31 & 32 & 9 & 19 & 29 & 39 \\
\hline & 1600 & 36 & 41 & 43 & 45 & 0 & 10 & 26 & 31 & 0 & 7 & 23 & 32 \\
\hline \multirow{5}{*}{0.80} & 100 & 39 & 41 & 44 & 46 & 9 & 22 & 30 & 32 & 18 & \begin{tabular}{|l|}
$\quad 29$ \\
\end{tabular} & \begin{tabular}{|l}
$\quad 40$ \\
\end{tabular} & 42 \\
\hline & 200 & 33 & 41 & 44 & 46 & 7 & 22 & 30 & 31 & 10 & 25 & 35 & 39 \\
\hline & 400 & 32 & 38 & 42 & 43 & 5 & 13 & 28 & 29 & 4 & $\begin{array}{ll}15 \\
1\end{array}$ & 27 & 36 \\
\hline & 800 & 30 & 35 & 40 & 42 & 1 & 5 & 23 & 28 & 0 & $\begin{array}{l}18 \\
18\end{array}$ & 21 & 29 \\
\hline & 1600 & 31 & 33 & 40 & 42 & 0 & 4 & 10 & 24 & 0 & 0 & 13 & 19 \\
\hline
\end{tabular}

specially for the smaller time limits. This somewhat unexpected result might be because of the pre-processing steps in the $\mathrm{B} \& \mathrm{C}$ algorithm.

\section{Summary and conclusion}

In this paper, we proposed a novel B\&B algorithm that solves the SAA-RCPSP in a much more efficient manner than the methods that are already existing in the literature. The goal in SAARCPSP is to select a subset of realizations, for which the optimal solution must be feasible, such that the resulting confidence level is at least $(1-\hat{\alpha})$. Instead of branching over realizations, we branch over casets of realizations and thus the complexity of the method is a function of the number of casets rather than a function of the number of realizations. If the activity realizations are discrete, then the number of casets usually is increased only slightly by increasing the number of the realizations.

In our experiments, we tested different priority rules for activities, based on which the B\&B tree is constructed. We noticed that, among several sorting criteria, sorting activities based on smaller total slack times significantly improves the performance of our B\&B algorithm. The performance analyses show that the efficiency of the B\&B algorithm is mainly due to the concepts of casets, since the number of casets increases moderately as we increase the number of realizations. The latter property, however, holds if the number of realizations is significantly larger than the numbers of activity modes.

We ran our B\&B algorithm together with a MILP formulation and a B\&C algorithm on benchmark instances with a size of 30 activities. The B\&B algorithm outperforms the MILP formulation and the $\mathrm{B} \& \mathrm{C}$ algorithm in terms of computational times as well as in terms of the number of solved instances within the time limit. 


\section{References}

Artigues, C., Michelon, P., and Reusser, S. Insertion techniques for static and dynamic resource-constrained project scheduling. European Journal of Operational Research, 149(2):249 - 267, 2003.

Artigues, C., Demassey, S., and Neron, E. Resource-Constrained Project Scheduling: Models, Algorithms, Extensions and Applications. Wiley, 2008.

Artigues, C., Leus, R., and Talla Nobibon, F. Robust optimization for resource-constrained project scheduling with uncertain activity durations. Flexible Services and Manufacturing Journal, 25(1-2):175-205, 2013.

Beraldi, P. and Ruszczyński, A. A branch and bound method for stochastic integer problems under probabilistic constraints. Optimization Methods and Software, 17(3):359-382, 2002.

Beraldi, P. and Ruszczyński, A. Beam search heuristic to solve stochastic integer problems under probabilistic constraints. European Journal of Operational Research, 167(1):35 - 47, 2005.

Blazewicz, J., Lenstra, J. K., and Rinnooy Kan, A. Scheduling subject to resource constraints: classification and complexity. Discrete Applied Mathematics, 5(1):11-24, 1983.

Bruni, M. E., Beraldi, P., Guerriero, F., and Pinto, E. A scheduling methodology for dealing with uncertainty in construction projects. Engineering Computations, 28:1064-1078, 2011a.

Bruni, M. E., Beraldi, P., Guerriero, F., and Pinto, E. A heuristic approach for resource constrained project scheduling with uncertain activity durations. Computers \& Operations Research, 38(9):1305-1318, 2011b.

Bruni, M. E., Beraldi, P., and Laganà, D. The express heuristic for probabilistically constrained integer problems. Journal of Heuristics, 19(3):423-441, 2013.

Bruni, M. E., Pugliese, L. D. P., Beraldi, P., and Guerriero, F. A two-stage stochastic programming model for the resource constrained project scheduling problem under uncertainty. In Proceedings of the 7th International Conference on Operations Research and Enterprise Systems - Volume 1: ICORES,, pages 194-200. INSTICC, SciTePress, 2018.

Bruni, M., Pugliese, L. D. P., Beraldi, P., and Guerriero, F. An adjustable robust optimization model for the resource-constrained project scheduling problem with uncertain activity durations. Omega, 71:66 $84,2017$.

Creemers, S. Minimizing the expected makespan of a project with stochastic activity durations under resource constraints. Journal of Scheduling, 18(3):263-273, 2015.

Davari, M. and Demeulemeester, E. The proactive and reactive resource-constrained project scheduling problem. Journal of Scheduling, 2017.

Deblaere, F., Demeulemeester, E., and Herroelen, W. Reactive scheduling in the multi-mode RCPSP. Computers \& Operations Research, 38(1):63-74, 2011a.

Deblaere, F., Demeulemeester, E., and Herroelen, W. Proactive policies for the stochastic resourceconstrained project scheduling problem. European Journal of Operational Research, 214(2):308-316, 2011b.

Demeulemeester, E. and Herroelen, W. New benchmark results for the resource-constrained project scheduling problem. Management Science, 43(11):1485-1492, 1997.

Demeulemeester, E. and Herroelen, W. Project Scheduling: A Research Handbook. Kluwer Academic Publisher, 2002.

Demeulemeester, E. and Herroelen, W. Robust project scheduling. Foundations and Trends in Technology, Information and Operations Management, 3:201-376, 2011.

Dentcheva, D., Prékopa, A., and Ruszczynski, A. Concavity and efficient points of discrete distributions in probabilistic programming. Mathematical Programming, 89(1):55-77, Nov 2000.

Klein, R. Scheduling of Resource Constrained Projects. Kluwer Academic Publisher, 2000.

Kolisch, R. and Sprecher, A. PSPLIB - a project scheduling problem library. European Journal of Operational Research, 96(1):205-216, 1997.

Koné, O., Artigues, C., Lopez, P., and Mongeau, M. Event-based MILP models for resource-constrained project scheduling problems. Computers $\&$ Operations Research, 38(1):3 - 13, 2011.

Küçükyavuz, S. On mixing sets arising in chance-constrained programming. Mathematical Programming, 132(1):31-56, 2012.

Lamas, P. and Demeulemeester, E. A purely proactive scheduling procedure for the resource-constrained project scheduling problem with stochastic activity durations. Journal of Scheduling, 19(4):409-428, 2016. 
Luedtke, J. A branch-and-cut decomposition algorithm for solving chance-constrained mathematical programs with finite support. Mathematical Programming, 146(1):219-244, 2014.

Luedtke, J. and Ahmed, S. A sample approximation approach for optimization with probabilistic constraints. SIAM Journal on Optimization, 19(2):674-699, 2008.

Luedtke, J., Ahmed, S., and Nemhauser, G. L. An integer programming approach for linear programs with probabilistic constraints. Mathematical Programming, 122(2):247-272, 2010.

Mingozzi, A., Maniezzo, V., Ricciardelli, S., and Bianco, L. An exact algorithm for the resource-constrained project scheduling problem based on a new mathematical formulation. Management Science, 44(5):714$729,1998$.

Möhring, R. and Radermacher, F. Introduction to stochastic scheduling problems. In Neumann, K. and Pallaschke, D., editors, Contributions to Operations Research, volume 240 of Lecture Notes in Economics and Mathematical Systems, pages 72-130. Springer Berlin Heidelberg, 1985.

Prékopa, A. Dual method for the solution of a one-stage stochastic programming problem with random rhs obeying a discrete probability distribution. Zeitschrift für Operations Research, 34(6):441-461, 1990.

Rostami, S., Creemers, S., and Leus, R. New benchmark results for the stochastic resource-constrained project scheduling problem. Technical report, KU Leuven, 2016.

Ruszczyński, A. Probabilistic programming with discrete distributions and precedence constrained knapsack polyhedra. Mathematical Programming, 93(2):195-215, 2002.

Sprecher, A. Scheduling resource-constrained projects competitively at modest memory requirements. Management Science, 46(5):710-723, 2000.

Stork, F. Stochastic resource-constrained project scheduling. PhD thesis, TU Berlin, 2001.

Stork, F. Branch-and-bound algorithms for stochastic resource-constrained project scheduling. Technical report, Technische Universitat Berlin, 2000.

Van de Vonder, S., Demeulemeester, E., Leus, R., and Herroelen, W. Perspectives in Modern Project Scheduling, chapter Proactive-Reactive Project Scheduling Trade-Offs and Procedures, pages 25-51. Springer US, Boston, MA, 2006.

Van de Vonder, S., Demeulemeester, E., and Herroelen, W. Proactive heuristic procedures for robust project scheduling: An experimental analysis. European Journal of Operational Research, 189(3):723-733, 2008.

Zheng, X., Wu, B., and Cui, X. Cell-and-bound algorithm for chance constrained programs with discrete distributions. European Journal of Operational Research, 260(2):421-431, 2017. 